\title{
Myeloid cell leukemia-1 is an important predictor of survival and progression of small cell lung cancer
}

\author{
Ting Zhou ${ }^{1 \#}$, Lin Zhang ${ }^{2 \#}$, Tingting Liu ${ }^{1 \#, ~ Y u n p e n g ~ Y a n g ~}{ }^{1}$, Fan Luo ${ }^{1}$, Zhonghan Zhang ${ }^{1}$, Yan Huang ${ }^{1}$, \\ Hongyun Zhao ${ }^{3}$, Li Zhang ${ }^{1}$, Yuanyuan Zhao ${ }^{1}$ \\ ${ }^{1}$ Department of Medical Oncology, Sun Yat-sen University Cancer Center, State Key Laboratory of Oncology in South China, Collaborative \\ Innovation Center for Cancer Medicine, Guangzhou, China; ${ }^{2}$ Department of Clinical Laboratory, Sun Yat-sen University Cancer Center, State \\ Key Laboratory of Oncology in South China, Collaborative Innovation Center for Cancer Medicine, Guangzhou, China; ${ }^{3}$ Department of Clinical \\ Research, Sun Yat-sen University Cancer Center, State Key Laboratory of Oncology in South China, Collaborative Innovation Center for Cancer \\ Medicine, Guangzhou, China \\ Contributions: (I) Conception and design: T Zhou, T Liu, Y Zhao; (II) Administrative support: L Zhang; (III) Provision of study materials or patients: \\ L Zhang; (IV) Collection and assembly of data: L Zhang, T Zhou; (V) Data analysis and interpretation: T Liu, T Zhou; (VI) Manuscript writing: All \\ authors; (VII) Final approval of manuscript: All authors. \\ \#These authors contributed equally to this work. \\ Correspondence to: Li Zhang; Yuanyuan Zhao. Department of Medical Oncology, Sun Yat-sen University Cancer Center, 651 Dongfeng Road East, \\ Guangzhou 510060, China. Email: zhangli@sysucc.org.cn; zhaoyy@sysucc.org.cn.
}

Background: Small cell lung cancer (SCLC) is the most fatal malignancy for which more effective therapies are urgently needed. Overexpression of myeloid cell leukemia-1 (Mcl-1) has been demonstrated to be one of the most common genetic alterations among different types of tumor/cancer, which induces resistance against various anti-cancer therapies including cisplatin. The study aimed to explore the role of Mcl-1 in the prognosis and resistance to anti-cancer therapy in patients with SCLC.

Methods: Patients with SCLC were recruited from those enrolled/treated in Sun Yat-sen University Cancer Center. Their specimens were collected for immunohistochemical evaluation. We compared the baseline characteristics, response to chemotherapy and overall survival (OS) of the patients with different expression levels of Mcl-1.

Results: The expression level of Mcl-1 was significantly lower in patients with limited stage SCLC than in those with extensive stage SCLC $(\mathrm{P}=0.014)$. Based on the median value of Mcl-1 expression level, the patients were divided into high and low Mcl-1 groups, respectively. Univariate analysis revealed that low Mcl-1 expression was associated with a significant improvement in OS, with a hazard ratio (HR) of 0.538. Multivariate analysis confirmed the independent prognostic value of Mcl-1 expression level $(\mathrm{P}=0.014)$. Moreover, we found a significantly close relationship between higher Mcl-1 expression level and shorter time to progression (TTP) of the patients received chemotherapy $(\mathrm{P}=0.040)$.

Conclusions: Our findings demonstrated that Mcl-1 expression level was a prognostic biomarker for survival outcomes and cancer progression in the patients with SCLC. Thus, it could be used as a valuable biomarker in identifying those patients with high risk of treatment failure.

Keywords: Myeloid cell leukemia-1 (Mcl-1); overall survival (OS); prognosis; small cell lung cancer (SCLC); time to progression (TTP)

Submitted Mar 08, 2020. Accepted for publication Sep 25, 2020.

doi: 10.21037/atm-20-2305

View this article at: http://dx.doi.org/10.21037/atm-20-2305 


\section{Introduction}

Lung cancer is one of the leading causes of cancer-related death in the world (1). Currently, approximately $15 \%$ of new cases of patients with lung cancer have been diagnosed as small cell lung cancer (SCLC) (2). SCLC behaves the most aggressively and is characterized by the rapid growth and high metastatic risk. A large proportion $(60 \%)$ of patients presents with extensive stage disease (ED-SCLC) $(3,4)$. Platinum-based chemotherapy still constitutes as the standard first-line care (4-6 cycles of etoposide plus either cisplatin or carboplatin) for patients with SCLC, particularly for those with ED-SCLC. Despite the high response rate of $65 \%$, most patients encounter with rapid progression and/or disease recurrence. The outcomes still remain very poor, with the median overall survival (OS) being shorter than 20 months in limited stage disease (LDSCLC) and only 12 months in ED-SCLC (5). Multiple clinical trials evaluating new chemotherapy agents or other treatment strategies have failed to improve the outcome significantly (6-9). Thus, further advances in the treatment of SCLC may rely on the development of novel classes of drugs to overcome the resistance of platinum-based drugs and/or of the newer targeted drugs targeting the related signal pathways.

Myeloid cell leukemia-1 (Mcl-1) is a unique member of anti-apoptosis B-cell lymphoma-2 (Bcl-2) family, with the functions in cell growth, tumorigenesis, and development of lymphocyte metastasis (10-13). Overexpression of Mcl-1 has been found in many types of human tumors, including breast cancer (14), melanoma (15), ovarian cancer (16), and non-small cell lung cancer (NSCLC) (17). Additionally, many lines of evidence have demonstrated that overexpression of Mcl-1 frequently induces the resistance to a number of the widely used anti-tumor drugs, including paclitaxel, gemcitabine, and especially, cisplatin (18-20). Inhibition of tumor growth and the increased cell apoptosis were observed in Mcl-1-overexpressing ovarian, lymphoma, colon cells, when Mcl-1 was knocked down. Furthermore, Yu et al. suggested that inhibition of Mcl-1 could enhance the cisplatin-induced apoptosis in esophageal squamous cell carcinoma cells (20), which was also verified in NSCLC cells (21). Depletion of Mcl-1 was reported to possibly enhance the sensitivity of NSCLC cells to cisplatin (22). Several studies reported that the overexpression of Mcl-1 was extremely and closely associated with inferior survival benefit of the patients with breast cancer $(23,24)$. Similarly, the analogic prognostic value of Mcl-1 was observed in
NSCLC (25). All together, these data indicate that Mcl1 can be a promising target for anti-cancer therapy. However, its clinical features and the role as a prognostic factor of Mcl-1 in SCLC remain unclear and need to be clarified. Therefore, we performed this retrospective study to assess the impact of Mcl-1 expression on OS and to explore the association between expression level of Mcl1 and the baseline clinicopathological characteristics and treatment response of the patients with SCLC, aiming to identify the potential patients with poorer prognosis and easier resistance to chemotherapy. Herein, we present the following article in accordance with the REMARK reporting checklist (available at http://dx.doi.org/10.21037/ atm-20-2305).

\section{Methods}

\section{Study population}

In this retrospective study, we screened a total of 80 patients with histologically or cytologically confirmed SCLC at the Sun Yat-sen University Cancer Center (SYSUCC) (Guangzhou, Guangdong, China) during the period between March 2008 and December 2013. The inclusion criteria were set as follows: (I) the availability of tissue specimens for immunohistochemical staining, and (II) the availability of eligible clinicopathological information. The patients who had multiple primary cancers or previous malignancies within 5 years were excluded. The study was conformed to the provisions of the Declaration of Helsinki (as revised in 2013) and approved by the Institutional Review Board of SYSUCC (ID: B2019-140-01). The written informed consent was obtained from each participant.

\section{Data extraction}

The pieces of information about the baseline clinicopathological features, including gender, age, smoking status, performance status (PS), cancer stage, and therapeutic data were collected. The smokers were defined as those who had smoked more than 100 cigarettes. The cancer staging was determined based on the Veteran Affairs Lung Study Group (VALG) staging system and the seventh edition of the American Joint Committee on Cancer (AJCC). The first-line chemotherapy consisted of cisplatin and etoposide was administered for most patients on day 1 throughout each cycle. Furthermore, the patients with LD- 


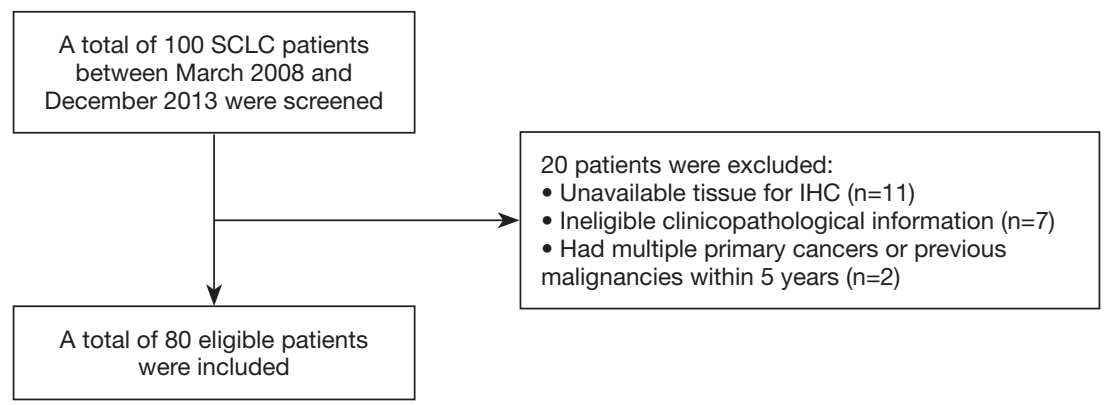

Figure 1 Flow chart of patients' enrollment. SCLC, small cell lung cancer.

SCLC even received concurrent chemoradiotherapy (CRT) or radical surgery.

\section{Follow-up}

All the enrolled patients were regularly followed up from pathological diagnosis to death. The last date of follow up was October 21, 2019. Tumor assessment was done every 2 cycles of chemotherapy and every 2 months after completion of treatment until the concurrence of disease progression, based on computerized tomography (CT) scanning. Tumor response to drugs was determined according to Response Evaluation Criteria in Solid Tumor $1.0(26)$.

\section{Immunobistochemistry (IHC) staining and scoring}

IHC staining was performed to assess Mcl-1 expression level. Tumor tissue samples were obtained from biopsy of the patients with SCLC and fixed with 10\% formalin for $24 \mathrm{~h}$, The paraffin-embedded tumor tissue slides ( $3 \mu \mathrm{m}$ thick) were coated with Mcl-1 antibody (dilution: 1:150), incubated at $37^{\circ} \mathrm{C}$ for $1 \mathrm{~h}$, labeled with horseradish peroxidase (HRP)labeled streptomycin affinity solution for $10 \mathrm{~min}$, and finally stained with diaminoaniline (DAB). Two pathologists independently determined the scores for IHC staining. The calculation formula of scores of Mcl-1 was as follows: staining intensity multiplied by percentage of positive cells in the staining area. The intensity was scored as follows: 0 , negative; 1 , weak; 2 , moderate; and 3 , strong. The frequency of positive cells was defined as follows: the percentage of positive tumor cells $<5 \%=0$, the percentage of positive tumor cells $<25 \%=1$, the percentage of positive tumor cells $<50 \%=2$, the percentage of positive tumor cells $<75 \%=3$, and the percentage of positive tumor cells $>75 \%=4$.

\section{Statistical analysis}

Continuous variables were changed into categorical variables based on their median values. Categorical variables were compared and evaluated with Chi-square or Fisher' exact test. The primary endpoint was OS, defined as the period from time of pathological diagnosis to the date of death for any cause or last follow-up. For the analysis of OS, the patients who were alive at the time of last contact were censored. OS rates were compared between groups using the unstratified logrank test, and hazard ratio (HR) and $95 \%$ confidence interval (95\% CI) were provided. Subgroup analysis was performed to identify the consistency of the prognostic value according to the baseline clinicopathological characteristics. Cox-proportional hazard model was used to conduct multivariate analysis of association between Mcl-1 expression level and other prognostic factors, which were assessed with logistic regression. A two-sided $\mathrm{P}$ value $\leq 0.05$ was considered statistically significant. All the statistical analyses were conducted via SPSS version 25.0 (IBM, Armonk, NY, USA).

\section{Results}

\section{Clinicopathological characteristics of patients}

A total of 80 SCLC patients with eligible tumor tissue samples and clinical information were included in this study (Figure 1). Median age of the enrolled patients at diagnosis was 59 years ranged from 22 to 78 years. Among them, 68 $(85 \%)$ were male and $12(15 \%)$ were female. A majority of them $(n=64,80 \%)$ were current or ever smokers who had a PS of $0-1(n=79,98.75 \%)$ and LD-SCLC $(n=50$, $62.5 \%$ ). In term of treatment, all the patients received platinum-based chemotherapy as the first-line treatment, 
most of them ( $\mathrm{n}=63,90 \%)$ received cisplatin. Their baseline clinicopathological characteristics are shown in Table 1.

\section{Association of Mcl-1 level with the clinicopathological characteristics}

Based on the median expression level of Mcl-1 of 8 ranged within $2-12$, the patients were divided into high (9-12, $\mathrm{n}=34)$ and low $(0-8, \mathrm{n}=46)$ expression groups, respectively (Figure 2). The patient's clinicopathological characteristics were described based on their Mcl-1 expression levels (Table 1). Compared with those with LD-SCLC, the patients with ED-SCLC had significantly higher expression level of Mcl-1 (P=0.014). Similarly, those received radical operation were more likely to have significantly lower Mcl-1 expression level $(\mathrm{P}=0.002)$. To further explore the relationship between Mcl-1 expression level and tumor stage, we analyzed the detailed Tumor, Node, Metastasis (TNM) classification. Interestingly, we found that the higher expression level of Mcl-1 was also significantly correlated with $\mathrm{M}$ classification $(\mathrm{P}=0.014)$, while there were no significant relations between $\mathrm{T} / \mathrm{N}$ classification and Mcl-1 level ( $\mathrm{P}=0.055$ and $\mathrm{P}=0.380$, respectively). Other clinicopathological characteristics, including gender, age, smoking status, PS score, chemotherapy regime, the received radiotherapy and progression sites, were similar between the patients with high Mcl-1 level and those with low Mcl-1 level.

\section{Association of Mcl-1 with time to progression (TTP)}

Of the 80 patients in the study, 38 patients suffered disease progression, and most of them received cisplatin-based chemotherapy as the first-line treatment. An exploratory analysis showed that Mcl-1 expression level was significantly correlated with TTP. An elevated Mcl-1 expression level was remarkably associated with shorter TTP (high Mcl-1 group vs. low Mcl-1 group: 5.75 vs. 6.70, $\mathrm{P}=0.04$ ) (Figure 3). Among them, in the patients with limited stage, we found a significantly consistent TTP benefit in the patients with low Mcl-1 expression level $(\mathrm{P}=0.018)$.

\section{Association of Mcl-1 level with OS}

The median follow-up for the entire population was 21.13 months ranged $0.30-121.49$. At the time of analysis, all the patients with high Mcl-1 expression level were alive whereas only one patient with low Mcl-1 expression level was alive. Their median OS was 12.67 months ranged within 0.20 107.1. In the univariate analysis, OS was significantly more closely associated with the patients in low Mcl-1 group than with those in high Mcl-1 group ( $\mathrm{P}=0.003$, Table 2, Figure $4 A$ ). Moreover, the results showed significant OS benefit in the patients with limited stage disease (16.26 vs. 9.36, $\mathrm{P}<0.001$, Table 2, Figure 4B). Nevertheless, no significant correlations between OS and age $(\mathrm{P}=0.360)$, between OS and gender $(\mathrm{P}=0.592)$ and between $\mathrm{OS}$ and smoking status

Table 1 Association between Mcl-1 expression level and the clinicopathological characteristics of the patients with SCLC

\begin{tabular}{|c|c|c|c|c|}
\hline Clinicopathological features & Total (\%) & \multicolumn{2}{|c|}{ Mcl-1, n (\%) } & $P$ value \\
\hline Gender & & & & 0.704 \\
\hline Female & $12(15.0)$ & $4(11.8)$ & $8(17.4)$ & \\
\hline Male & $68(85.0)$ & $30(88.2)$ & $38(82.6)$ & \\
\hline$\leq 59$ & $41(51.3)$ & $16(47.1)$ & $25(54.3)$ & \\
\hline$>59$ & $39(48.7)$ & $18(52.9)$ & $21(45.7)$ & \\
\hline Smoking status & & & & 0.497 \\
\hline Current or ever & $64(80.0)$ & $26(76.5)$ & $38(82.6)$ & \\
\hline
\end{tabular}

Table 1 (continued) 
Table 1 (continued)

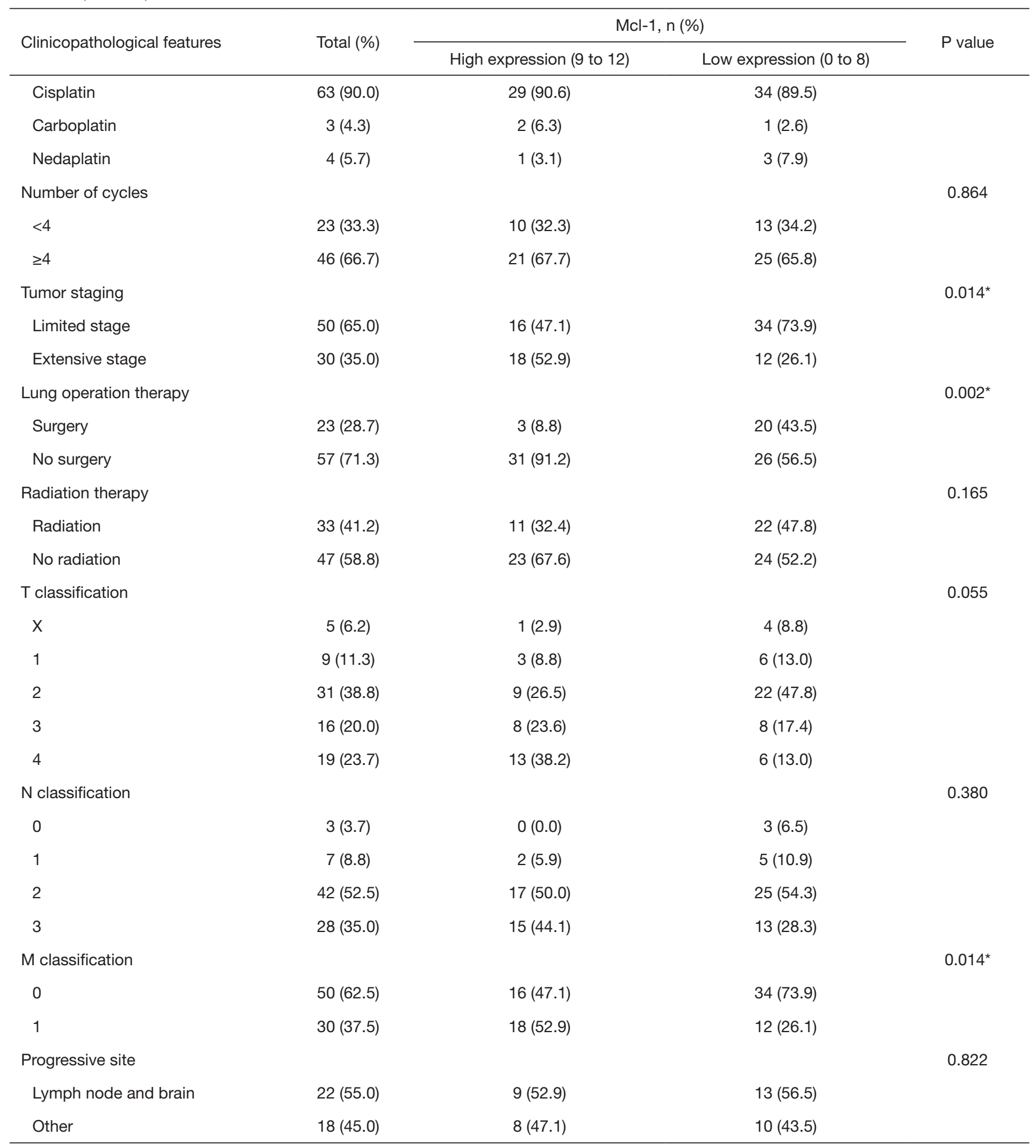

${ }^{*}, \mathrm{P}<0.05$. Mcl-1, myeloid cell leukemia-1; SCLC, small cell lung cancer. 

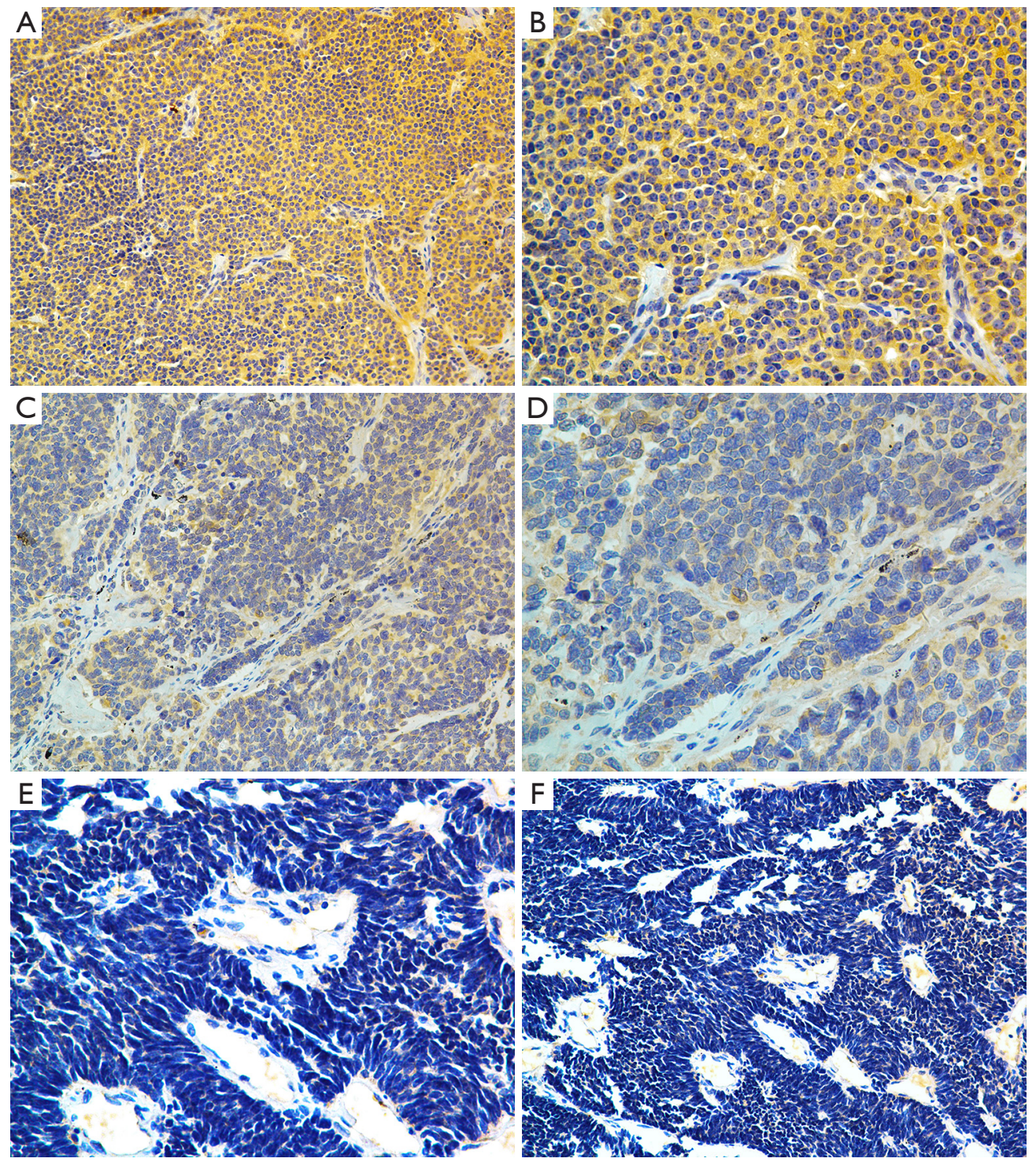

Figure 2 Representative images of immunostaining of Mcl-1 expression with different scores. (A) High expression level of Mcl-1 (magnification, ×20); (B) high expression level of Mcl-1 (magnification, ×40); (C) low expression level of Mcl-1 (magnification, ×20); (D) low expression level of Mcl-1 (magnification, $\times 40$ ); (E) the negative expression level of Mcl-1 (magnification, $\times 20)$; (F) the high expression level of Mcl-1 (magnification, $\times 40$ ). The tissue sections were dewaxed in xylene, rehydrated, and rinsed in graded ethanol solutions. The antigens were retrieved by heating the tissue sections at $100{ }^{\circ} \mathrm{C}$ for $5 \mathrm{~min}$ in EDTA $(1 \mathrm{mmol} / \mathrm{L}, \mathrm{pH} 9.0)$ solution when necessary, and then immersed in a $0.3 \%$ hydrogen peroxide solution for $10 \mathrm{~min}$ to block endogenous peroxidase activity, rinsed in phosphate buffered saline (PBS) for 5 min, and incubated with the MCL-1 primary antibody (1:100 dilution, CST\#39224; Cell Signaling Technology) at $4{ }^{\circ} \mathrm{C}$ overnight. The slides were washed with $1 \times$ PBS and treated with a goat antibody against a rabbit secondary antibody (EnVision; Dako, Glostrup, Denmark) at $37.5^{\circ} \mathrm{C}$ for $30 \mathrm{~min}$. Finally, the visualized staining was developed with 3,3'-diaminobenzidine tetrahydrochloride (DAB, Dako, Glostrup, Denmark), and all of the slides were counterstained with hematoxylin. Mcl-1, myeloid cell leukemia-1. 
$(\mathrm{P}=0.606)$ were observed (Table 2).

The benefit on OS in low Mcl-1 expression group was verified in the multivariate analyses (Table 3). The Mcl-1 expression level appeared to be an independent prognostic factor for OS. As compared to the group with high Mcl-1 expression level, the group with low Mcl-1 expression level had 0.538 -fold lower risk of death (HR 0.538; 95\% CI: $0.366-0.981 ; \mathrm{P}=0.042$ ).

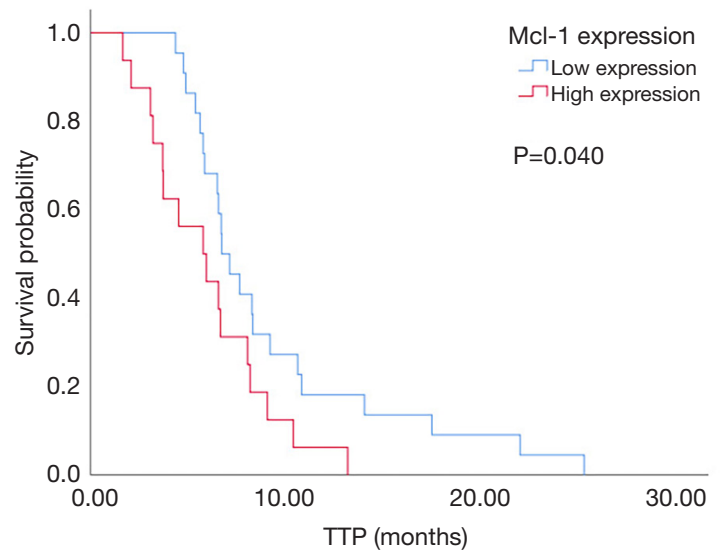

Figure 3 Kaplan-Meier survival curves comparing TTP: low Mcl1 expression level $v s$. high Mcl-1 expression level. Mcl-1, myeloid cell leukemia-1; TTP, time to progression.

\section{Discussion}

To the best of our knowledge, this is the first study evaluating the prognostic power of Mcl-1 in patients with SCLC. Our findings show that the patients with high expression level of Mcl-1 have a significantly lower OS as compared with that of the patients having low expression level of Mcl-1. Multivariable analysis has demonstrated that the lower expression level of $\mathrm{Mcl}-1$ is independently associated with longer OS. In recent years, accumulating lines of evidence have suggested that overexpression of Mcl-1 is the most common genetic alterations in several types of human cancers, including breast cancer, ovarian cancer and melanoma $(15,16,27)$. Similarly, Mcl-1 has been regularly found to be overexpressed in lung cancer cell lines $(17,28,29)$. Data about the prognostic value of Mcl-1 have been emerging, with the results indicating that Mcl-1 is closely related to the survival benefit in the patients with breast, pancreatic, and gastric cancer $(10,30,31)$. Furthermore, the beneficial prognostic role of Mcl-1 in clinical outcomes for the patients with non-small lung cancer cells (NSCLC) has also been verified in some researches (32-34). The results reported in the current study are in accordance with the outcomes reported in these previous studies regarding NSCLC patients, with a similar prognostic power that $\mathrm{Mcl}-1$ is significantly associated with poor prognosis (high Mcl-1 group vs. low Mcl-1 group:

Table 2 Univariate analysis of OS

\begin{tabular}{|c|c|c|c|c|}
\hline Variable & Cases number & Proportion (\%) & OS (months), median (95\% Cl) & $P$ value \\
\hline Gender & & & & 0.592 \\
\hline Female & 12 & 15.00 & $11.79(4.76-18.82)$ & \\
\hline Male & 68 & 85.00 & $12.81(9.29-16.34)$ & \\
\hline No & 16 & 20.00 & 11.79 (3.03-20.55) & \\
\hline Yes & 64 & 80.00 & $12.81(9.81-15.81)$ & \\
\hline Expression level of Mcl-1 & & & & $0.003^{*}$ \\
\hline Low & 46 & 57.50 & $16.26(12.23-20.30)$ & \\
\hline Limited stage & 50 & 62.5 & $16.26(12.75-19.77)$ & \\
\hline Extensive stage & 30 & 37.5 & $9.36(6.80-11.92)$ & \\
\hline
\end{tabular}

*, $\mathrm{P}<0.05$. Mcl-1, myeloid cell leukemia-1; OS, overall survival; $\mathrm{Cl}$, confidence interval. 

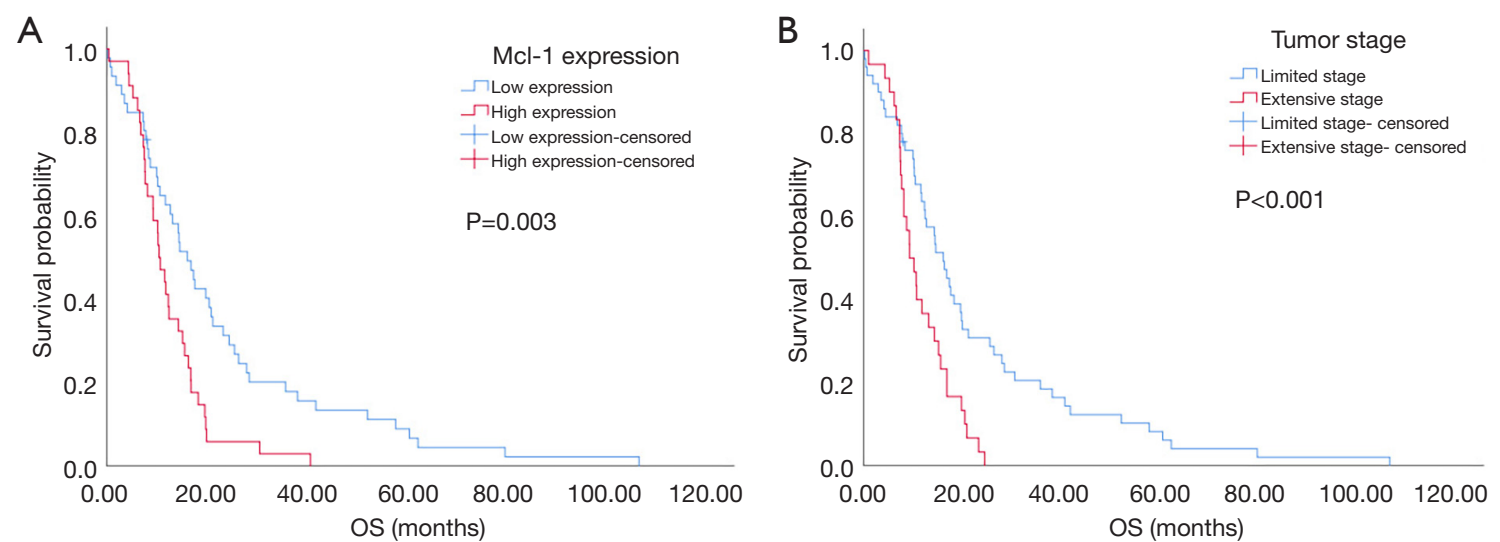

Figure 4 Kaplan-Meier survival curves comparing OS. (A) Low Mcl-1 expression vs. high Mcl-1 expression; (B) limited stage vs. extensive stage. OS, overall survival; Mcl-1, myeloid cell leukemia-1.

Table 3 Cox regression model analysis

\begin{tabular}{lccc}
\hline Variable & P value & $\mathrm{HR}$ & \multicolumn{1}{c}{$95 \% \mathrm{Cl}$} \\
\hline Expression of Mcl-1 (score) & 0.014 & 0.538 & $0.328-0.882$ \\
Tumor staging & 0.008 & 0.500 & $0.300-0.833$ \\
\hline
\end{tabular}

Mcl-1, myeloid cell leukemia-1; HR, hazard ratio; $\mathrm{Cl}$, confidence interval.

8.48-12.62 vs.12.23-20.30, $\mathrm{P}=0.003)$. Mcl-1 is a virtual anti-apoptotic protein and exerts its anti-apoptotic power to promote tumor development and progression (12). While the tumorigenesis mechanism of Mcl-1 in SCLC was unclear, its anti-apoptotic function may be explained by our results showing that Mcl-1 expression is negatively associated with OS in SCLC patients. Another underlying mechanism might be related to the direct interaction of Mcl-1 with AKT at the pleckstrin homology $(\mathrm{PH})$ domain (35), which plays a significant role in maintaining AKT in an inactive state. Moreover, Hong et al. also found that Mcl-1 was significantly associated with AKT/mTOR pathway (36). Thus, targeting the mTOR/Mcl-1 axis can be effective against SCLC.

According to the NCCN guideline, currently, the standard first-line chemotherapy for SCLC patients is still cisplatin-based regime. Therefore, we analyzed the relation between Mcl-1 and TTP in the patients received the cisplatin-containing first-line chemotherapy. Our results revealed a close correlation of Mcl-1 with risk of progression in the patients with SCLC. The patients with high Mcl-1 expression level was associated with lower TTP, as compared with those with low expression level of Mcl-1, especially in the patients in the limited stage (high Mcl-1 group vs. low Mcl-1 group: 2.981-8.518 vs. 5.456-7.948, $\mathrm{P}=0.04)$. A number of studies have shown that overexpression of Mcl-1 can contribute to the resistance of multiple types of cancer to cisplatin $(19,20,37)$. Additionally, the in vitro studies have also suggested that inhibition of Mcl-1 activity by specific inhibitors can enhance the sensibility to cisplatin and increase the apoptosis of lung cancer cells after receiving cisplatin (22). Our investigation suggested that overexpression of Mcl-1 could induce the cisplatin-resistance of SCLC. Lines of emerging evidence have indicated that as a novel member in the Bcl-2 family, Mcl-1 plays a critical role in chemotherapy resistance $(38,39)$. One of the potential mechanisms might be the association between Mcl-1 and TGF- $\beta$-induced epithelialto-mesenchymal transition (EMT) being considered as an essential process in the chemo-resistance of NSCLC (40). Therefore, further studies with focus on elucidating these mechanisms need to be conducted. Moreover, our findings are also of clinical significance. We could stratify the patients with high or low possibility of cisplatin-resistance by examining their expression levels of Mcl-1. In clinical practice, if the patients with SCLC have high expression level of Mcl-1, they could be considered to have high risk of cisplatin-resistance or tumor progression after the 
first-line treatment and thus, more frequent follow-up is recommended. In addition, a Mcl-1 inhibitor could be used in combination with the cisplatin-based chemotherapy to reduce the cisplatin resistance. However, the patients with low Mcl-1 expression levels are likely to have low risk of cisplatin-resistance, and they may acquire more benefit from chemotherapy. For these patients, regular follow-up is preferred. Mcl-1 is essential for initial tumor development and progression, as well as for the metastasis of lymphocytes and neurons $(13,41)$. The results of our study had validated these hypotheses from another prospective. Our findings have demonstrated that Mcl-1 expression level is significantly associated with cancer stage. Compared with the patients with high expression levels of Mcl-1, those patients with low expression levels of Mcl-1 are significantly likely in limited stage and their probability of receiving radical operation can be higher.

However, there are several limitations for the present study. First, this is a retrospective and singlecentered study, and thus, it may have the potential selection bias. To minimize this bias, we have identified the consecutive patients, and after adjustment for basic clinical characteristics, Mcl-1 also acts as the independent prognostic factor. Second, nowadays, the addition of programmed cell death ligand 1 (PD-L1) antibody to chemotherapy has become a new regime for the first-line treatment of SCLC patients. Whether Mcl-1 can serve as the prognostic factor for the patients receiving combined immunotherapy and chemotherapy is still needed to be further explored. Certainly, prospective, large-scale, and multiple-centered studies are needed to further verify our findings.

\section{Conclusions}

In conclusion, this study has demonstrated that lower expression level of Mcl-1 is an independent prognostic biomarker for longer OS in SCLC patients. Moreover, the results of TTP analysis, which are consistent with those reported in the previous in vitro studies, indicate that lower Mcl-1 expression level is also associated with better TTP. Therefore, the patients with higher Mcl-1 expression level may be at an increased risk of failure and death, and more aggressive therapy regime should be considered.

\section{Acknowledgments}

Funding: This work was supported by National Key
R\&D Program of China (2016YFC0905500 and 2016YFC0905503), Science and Technology Program of Guangdong (2017B020227001), Science and Technology Program of Guangzhou (201607020031), Chinese National Natural Science Foundation Project (81772476), the Natural Science Foundation of Guangdong Province of China (2018A030313838) and the 5010 Clinical Research Foundation of Sun Yat-sen University (2016001). All the grant supporting agencies have no roles in study design, data collection and analysis, and manuscript preparation.

\section{Footnote}

Reporting Checklist: The authors have completed the REMARK reporting checklist. Available at http://dx.doi. org/10.21037/atm-20-2305

Data Sharing Statement: Available at http://dx.doi. org/10.21037/atm-20-2305

Conflicts of Interest: All authors have completed the ICMJE uniform disclosure form (available at http://dx.doi. org/10.21037/atm-20-2305). The authors have no conflicts of interest to declare.

Ethical Statement: The authors are accountable for all aspects of the work in ensuring that questions related to the accuracy or integrity of any part of the work are appropriately investigated and resolved. The study was conformed to the provisions of the Declaration of Helsinki (as revised in 2013) and approved by the Institutional Review Board of SYSUCC (ID: B2019-140-01). The written informed consent was obtained from each participant.

Open Access Statement: This is an Open Access article distributed in accordance with the Creative Commons Attribution-NonCommercial-NoDerivs 4.0 International License (CC BY-NC-ND 4.0), which permits the noncommercial replication and distribution of the article with the strict proviso that no changes or edits are made and the original work is properly cited (including links to both the formal publication through the relevant DOI and the license). See: https://creativecommons.org/licenses/by-nc-nd/4.0/.

\section{References}

1. Siegel RL, Miller KD, Jemal A. Cancer statistics, 2019. 
CA Cancer J Clin 2019;69:7-34.

2. Herbst RS, Heymach JV, Lippman SM. Lung cancer. N Engl J Med 2008;359:1367-80.

3. Byers LA, Rudin CM. Small cell lung cancer: where do we go from here? Cancer 2015;121:664-72.

4. van Meerbeeck JP, Fennell DA, De Ruysscher DK. Smallcell lung cancer. Lancet 2011;378:1741-55.

5. Lally BE, Urbanic JJ, Blackstock AW, et al. Small cell lung cancer: have we made any progress over the last 25 years? Oncologist 2007;12:1096-104.

6. Sanborn RE, Patel JD, Masters GA, et al. A randomized, double-blind, phase 2 trial of platinum therapy plus etoposide with or without concurrent vandetanib (ZD6474) in patients with previously untreated extensive-stage small cell lung cancer: Hoosier Cancer Research Network LUN06-113. Cancer 2017;123:303-11.

7. Oze I, Hotta K, Kiura K, et al. Twenty-seven years of phase III trials for patients with extensive disease smallcell lung cancer: disappointing results. PLoS One 2009;4:e7835.

8. Pujol JL, Lavole A, Quoix E, et al. Randomized phase II-III study of bevacizumab in combination with chemotherapy in previously untreated extensive small-cell lung cancer: results from the IFCT-0802 trialdagger. Ann Oncol 2015;26:908-14.

9. Murray N, Turrisi AT 3rd. A review of first-line treatment for small-cell lung cancer. J Thorac Oncol 2006;1:270-8.

10. Lee WS, Park YL, Kim N, et al. Myeloid cell leukemia-1 regulates the cell growth and predicts prognosis in gastric cancer. Int J Oncol 2015;46:2154-62.

11. Craig RW. MCL1 provides a window on the role of the BCL2 family in cell proliferation, differentiation and tumorigenesis. Leukemia 2002;16:444-54.

12. Thomas LW, Lam C, Edwards SW. Mcl-1; the molecular regulation of protein function. FEBS Lett 2010;584:2981-9.

13. Dzhagalov I, Dunkle A, He YW. The anti-apoptotic Bcl-2 family member Mcl-1 promotes T lymphocyte survival at multiple stages. J Immunol 2008;181:521-8.

14. Ding Q, He X, Xia W, et al. Myeloid cell leukemia-1 inversely correlates with glycogen synthase kinase-3beta activity and associates with poor prognosis in human breast cancer. Cancer Res 2007;67:4564-71.

15. Boisvert-Adamo K, Longmate W, Abel EV, et al. Mcl-1 is required for melanoma cell resistance to anoikis. Mol Cancer Res 2009;7:549-56.

16. Brotin E, Meryet-Figuiere M, Simonin K, et al. Bcl$\mathrm{XL}$ and MCL-1 constitute pertinent targets in ovarian carcinoma and their concomitant inhibition is sufficient to induce apoptosis. Int J Cancer 2010;126:885-95.

17. Luo L, Zhang T, Liu H, et al. MiR-101 and Mcl-1 in non-small-cell lung cancer: expression profile and clinical significance. Med Oncol 2012;29:1681-6.

18. Wertz IE, Kusam S, Lam C, et al. Sensitivity to antitubulin chemotherapeutics is regulated by MCL1 and FBW7. Nature 2011;471:110-4.

19. Ding Q, He X, Hsu JM, et al. Degradation of Mcl-1 by beta-TrCP mediates glycogen synthase kinase 3 -induced tumor suppression and chemosensitization. Mol Cell Biol 2007;27:4006-17.

20. Yu X, Li W, Xia Z, et al. Targeting MCL-1 sensitizes human esophageal squamous cell carcinoma cells to cisplatin-induced apoptosis. BMC Cancer 2017;17:449.

21. Li J, Viallet J, Haura EB. A small molecule pan-Bcl-2 family inhibitor, GX15-070, induces apoptosis and enhances cisplatin-induced apoptosis in non-small cell lung cancer cells. Cancer Chemother Pharmacol 2008;61:525-34.

22. Whitsett TG, Mathews IT, Cardone MH, et al. Mcl1 mediates TWEAK/Fn14-induced non-small cell lung cancer survival and therapeutic response. Mol Cancer Res 2014;12:550-9.

23. Campbell KJ, Dhayade S, Ferrari N, et al. MCL-1 is a prognostic indicator and drug target in breast cancer. Cell Death Dis 2018;9:19.

24. O'Driscoll L, Cronin D, Kennedy SM, et al. Expression and prognostic relevance of Mcl-1 in breast cancer. Anticancer Res 2004;24:473-82.

25. Wen Q, Zhan Y, Zheng H, et al. Elevated expression of mcl-1 inhibits apoptosis and predicts poor prognosis in patients with surgically resected non-small cell lung cancer. Diagn Pathol 2019;14:108.

26. Therasse P, Arbuck SG, Eisenhauer EA, et al. New guidelines to evaluate the response to treatment in solid tumors. European Organization for Research and Treatment of Cancer, National Cancer Institute of the United States, National Cancer Institute of Canada. J Natl Cancer Inst 2000;92:205-16.

27. Aliabadi HM, Mahdipoor P, Uludag H. Polymeric delivery of siRNA for dual silencing of Mcl-1 and P-glycoprotein and apoptosis induction in drug-resistant breast cancer cells. Cancer Gene Ther 2013;20:169-77.

28. Zhang H, Guttikonda S, Roberts L, et al. Mcl-1 is critical for survival in a subgroup of non-small-cell lung cancer cell lines. Oncogene 2011;30:1963-8.

29. Zarogoulidis P, Petanidis S, Kioseoglou E, et al. MiR- 
205 and miR-218 expression is associated with carboplatin chemoresistance and regulation of apoptosis via Mcl-1 and Survivin in lung cancer cells. Cell Signal 2015;27:1576-88.

30. Williams MM, Lee L, Hicks DJ, et al. Key Survival Factor, Mcl-1, Correlates with Sensitivity to Combined Bcl-2/BclxL Blockade. Mol Cancer Res 2017;15:259-68.

31. Samm N, Werner K, Ruckert F, et al. The role of apoptosis in the pathology of pancreatic cancer. Cancers (Basel) 2010;3:1-16.

32. Belmar J, Fesik SW. Small molecule Mcl-1 inhibitors for the treatment of cancer. Pharmacol Ther 2015;145:76-84.

33. Oh BS, Shin EA, Jung JH, et al. Apoptotic Effect of Galbanic Acid via Activation of Caspases and Inhibition of Mcl-1 in H460 Non-Small Lung Carcinoma Cells. Phytother Res 2015;29:844-9.

34. Wang T, Yang Z, Zhang Y, et al. Caspase cleavage of Mcl-1 impairs its anti-apoptotic activity and proteasomal degradation in non-small lung cancer cells. Apoptosis 2018;23:54-64.

35. Chen G, Park D, Magis AT, et al. Mcl-1 Interacts with Akt to Promote Lung Cancer Progression. Cancer Res

Cite this article as: Zhou T, Zhang L, Liu T, Yang Y, Luo F, Zhang Z, Huang Y, Zhao H, Zhang L, Zhao Y. Myeloid cell leukemia-1 is an important predictor of survival and progression of small cell lung cancer. Ann Transl Med 2020;8(23):1589. doi: 10.21037/atm-20-2305
2019;79:6126-38.

36. Hong B, Wang H, Deng K, et al. Combination treatment of RAD001 and BEZ235 exhibits synergistic antitumor activity via down-regulation of $\mathrm{p}-4 \mathrm{E}-\mathrm{BP} 1 / \mathrm{Mcl}-1$ in small cell lung cancer. Oncotarget 2017;8:106486-98.

37. Yin $W$, Nie $Y$, Zhang Z, et al. miR-193b acts as a cisplatin sensitizer via the caspase-3-dependent pathway in HCC chemotherapy. Oncol Rep 2015;34:368-74.

38. Michels J, Obrist F, Vitale I, et al. MCL-1 dependency of cisplatin-resistant cancer cells. Biochem Pharmacol 2014;92:55-61.

39. Ma J, Zhao Z, Wu K, et al. MCL-1 is the key target of adjuvant chemotherapy to reverse the cisplatin-resistance in NSCLC. Gene 2016;587:147-54.

40. Toge M, Yokoyama S, Kato S, et al. Critical contribution of MCL-1 in EMT-associated chemo-resistance in A549 non-small cell lung cancer. Int J Oncol 2015;46:1844-8.

41. Arbour N, Vanderluit JL, Le Grand JN, et al. Mcl-1 is a key regulator of apoptosis during CNS development and after DNA damage. J Neurosci 2008;28:6068-78. 